\title{
Metastatic renal cell carcinoma to the testis: A case report and review of the literature
}

\author{
Karim Marzouk, MD; Fahad Alyami, MD; ${ }^{*}$ Jennifer Merrimen, MD; ${ }^{*}$ Scott Bagnell, $M D^{*}$ \\ *Department of Urology, Dalhousie University, Halifax, NS; †Department of Pathology, Dalhousie University, Halifax, NS
}

Cite as: Can Urol Assoc J 2014;8(11-12):e924-7. http://dx.doi.org/10.5489/cuaj.2209 Published online December 15, 2014.

\section{Abstract}

This is a case of a 68-year-old male who presented with a chief compliant of a testicular mass, which was discovered to be a metastatic lesion of undiagnosed renal cell carcinoma. A computed tomography scan revealed a large right renal mass and multiple pulmonary metastasis. Shortly after diagnosis, the patient was initiated on systemic therapy and received a cytoreductive nephrectomy. We discuss the details of this case as well as a pertinent review of metastatic renal cell carcinoma to the testes.

\section{Introduction}

Renal cell carcinoma (RCC) is a lethal tumour that accounts for about $3 \%$ of all adult malignancies. ${ }^{1}$ Metastatic spread of RCC occurs through hematogenous and lymphatic spread of tumour cells to distant sites, often in an unpredictable manner due to the varying lymphatic drainage of the kidney. Typically, the most common sites of metastatic RCC are lung, bone, regional lymph nodes, liver, and brain. About $25 \%$ to $30 \%$ of patients will present with metastatic disease at the time of diagnosis. ${ }^{2}$ Of those with organ-confined disease who undergo nephrectomy, 20\% will relapse with recurrent RCC. ${ }^{3,4}$ Testicular metastasis from RCC is extremely rare. Because of the paucity of literature on the topic, little is known with regards to the patterns of spread and the association between metastatic RCC and dissemination to the testes. We present our own case of metastatic RCC masking as a primary testicular tumour, and discuss previous reports of similar presentations.

\section{Case report}

A 68-year-old man was referred to our urology clinic. He had a left-sided testicular mass, in addition to right-sided abdominal fullness. The patient believed that he first detected the testicular lump about 6 months before coming to the clinic and that it had been continuing to grow, and within the last 2 to 3 months also noticed a right abdominal bulge. Overall his symptoms were remarkable for progressive shortness of breath on exertion over the last 2 to 3 months. He denied any history of abdominal or flank pain, lower urinary tract symptoms, hematuria, recent weight loss, or hemoptysis. His medical history was significant only for hypertension, psoriasis, osteoarthritis, and a remote history of smoking.

On examination the patient demonstrated a 3- to $4-\mathrm{cm}$ fixed mass at the cranial aspect of the left testicle and he was slightly tender on palpation. The contralateral testicle was normal and there was no palpable inguinal adenopathy. There was an appreciable mass overlying the right abdominal flank area, otherwise his abdominal exam was unremarkable. He did not have any palpable supraclavicular nodes.

A scrotal ultrasound revealed a hypervascular soft tissue mass, measuring $3.5 \mathrm{~cm}$, within the left testicle (Fig. 1). Additionally, a computed tomography (CT) scan demonstrated a $12.2-\mathrm{cm}$ mass originating from the medial inferior aspect of the right kidney, with no obvious renal vein, inferior vena cava, adrenal, or retroperitoneal involvement (Fig.2, Fig. 3). However, there were multiple bilateral lung lesions ranging in size from $2 \mathrm{~mm}$ to $2 \mathrm{~cm}$ (Fig. 4). Preoperative blood work overall was unremarkable, except for some degree of anemia, hemoglobin of $104 \mathrm{~g} / \mathrm{L}$, and a mildly elevated alkaline phosphatase (ALP) of $122 \mathrm{U} / \mathrm{L}$. All other tumour markers were negative, including alpha fetoprotein (1 ug/L), beta HCG $(<1 \mathrm{IU} / \mathrm{L})$, and lactate dehydrogenase (94 U/L). Bone scintigraphy was performed because of the mildly elevated ALP, however this failed to demonstrate any metastatic bone lesions.

The original clinical impression was that this patient likely had 2 independent primary tumours, with a right RCC and an independent left testicular tumour. Subsequently, he was taken to the operating room for a left radical orchiectomy. Pathology results from the orchiectomy revealed RCC, clear cell type with a Fuhrman nuclear grade 4. 


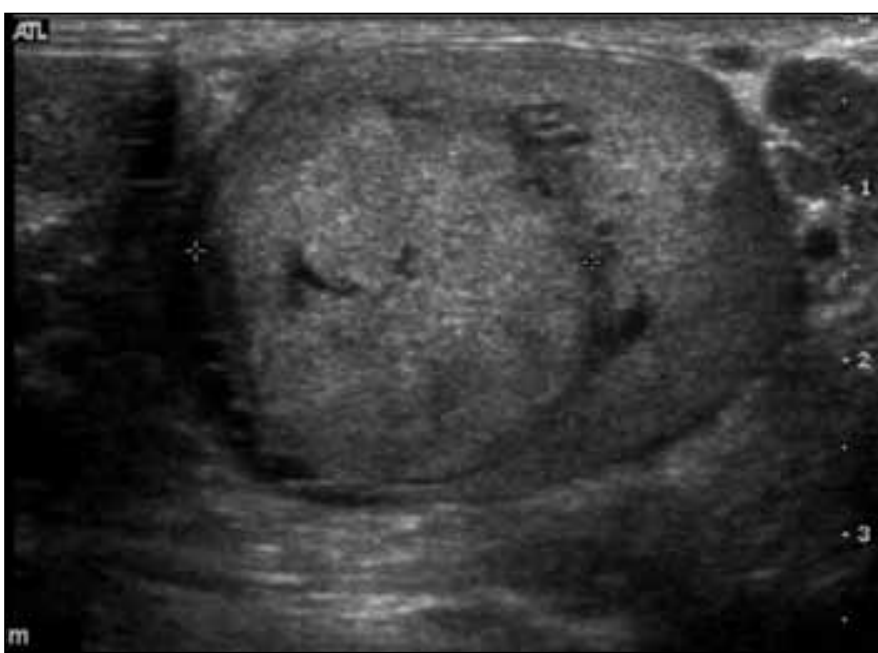

Fig. 1. Left testicular mass measuring $3.5 \times 2.5 \times 2.3 \mathrm{~cm}$.

Following the pathology results, the patient was referred to medical oncology and was initiated on systemic therapy with sunitinib. He received a total of 3 cycles prior to undergoing a cytoreductive right nephrectomy, and ultimately was diagnosed with pT3aNxM1 (stage IV) RCC. He recovered uneventfully from surgery, however he did experience side effects from systemic therapy in the form of skin blisters, oral and nasal mucosal ulcers. A follow-up CT revealed interval enlargement of his metastatic lung lesions 3 months after surgery (Fig. 5). Due disease progression, sunitinib was discontinued and he was enrolled in a clinical trial on atixinib. His most recent imaging has shown regression of the lung lesions and no other recurrences. Aside from ongoing respiratory symptoms, the patient is doing well and is tolerating systemic therapy.

\section{Pathologic findings}

The specimen consisted of a testicle, spermatic cord, and adjacent tissue in keeping with a radical orchiectomy. On cut section, the testicle contains a well-circumscribed yellow and hemorrhagic mass measuring $3.5 \times 2.4 \times 2.4 \mathrm{~cm}$ (Fig. 6). The lesion was confined to the testicle with no involvement of the tunicas, paratesticular soft tissue, or spermatic cord.

Microscopically, the tumour had a small nested or alveolar growth pattern and possessed a fine capillary network in the background. The cells possessed abundant clear to pale eosinophilic cytoplasm. Nuclei showed prominent nucleoli and areas of nuclear pleomorphism in keeping with a Fuhrman nuclear grade 4 . The tumour cells coexpressed monokeratin and vimentin and were CD10 positive. Cytokeratin 7 immunostain was negative (Fig. 7). These features were in keeping with a metastatic clear cell RCC.

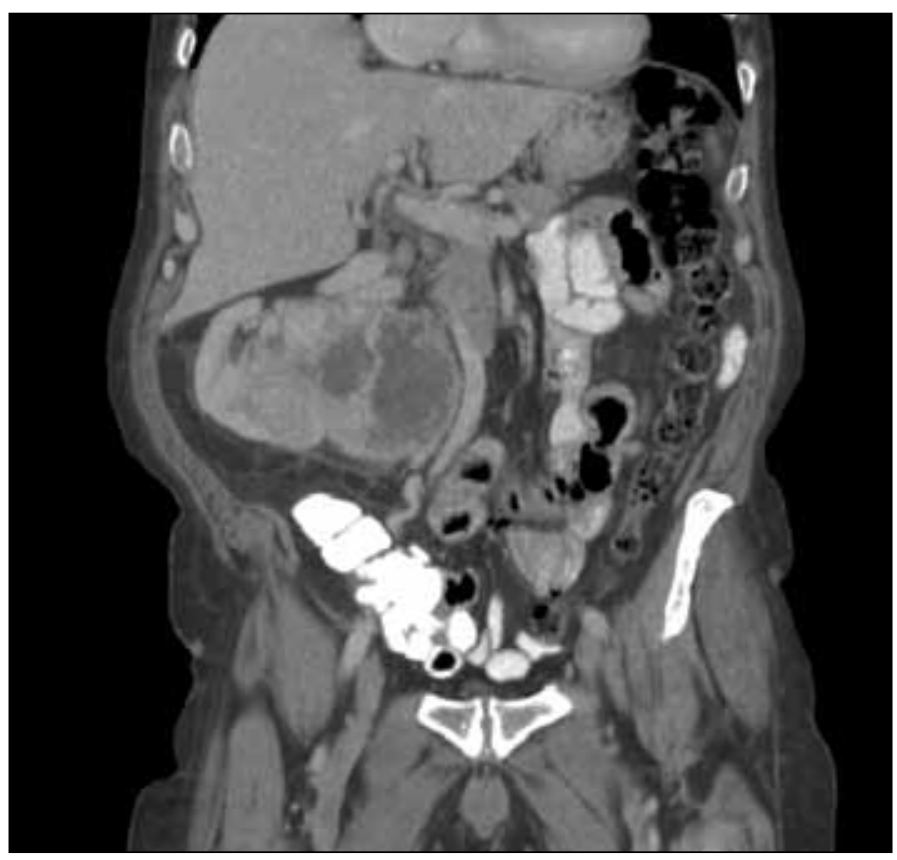

Fig. 2. Right renal mass, heterogenous measuring $12.2 \times 8.1 \mathrm{~cm}$.

\section{Discussion}

Metastatic disease to the testes is uncommon, with an estimated incidence rate of $0.3 \%$ to $3.6 \% .^{5}$ Historically, the most common source of metastasis identified has been from prostate cancer; however, there have been previous reports of RCC spread to the testicle. ${ }^{6}$ In our review of the literature, we identified 27 cases of metastatic RCC to the testes in the English language, and in all except for one, they were clear cell carcinomas. ${ }^{5-11}$ In most cases, patients were between the sixth and seventh decade, ${ }^{5,7-9}$ although in one publica-

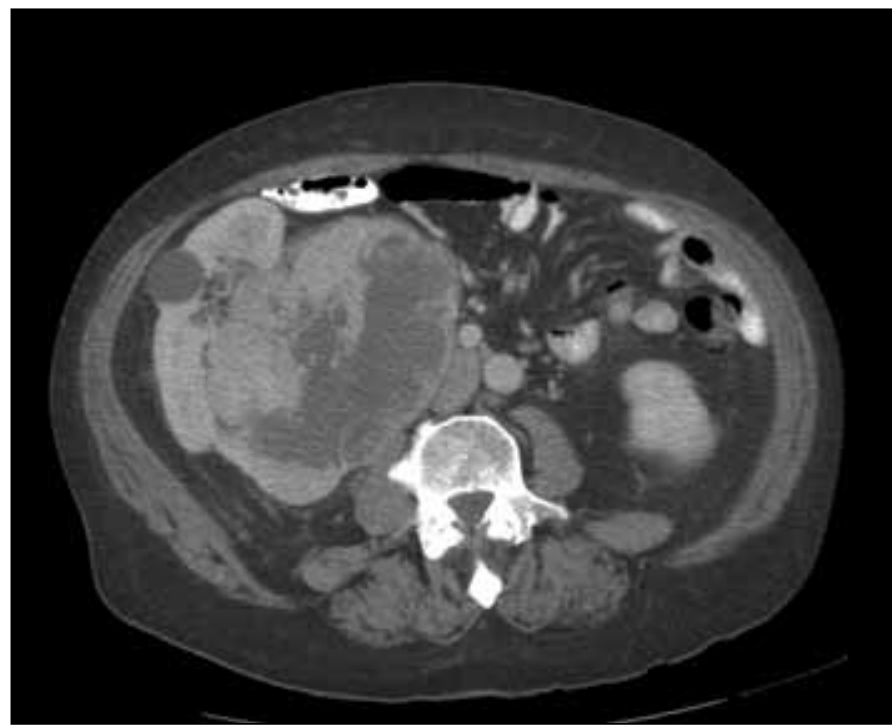

Fig. 3. Right renal mass. 


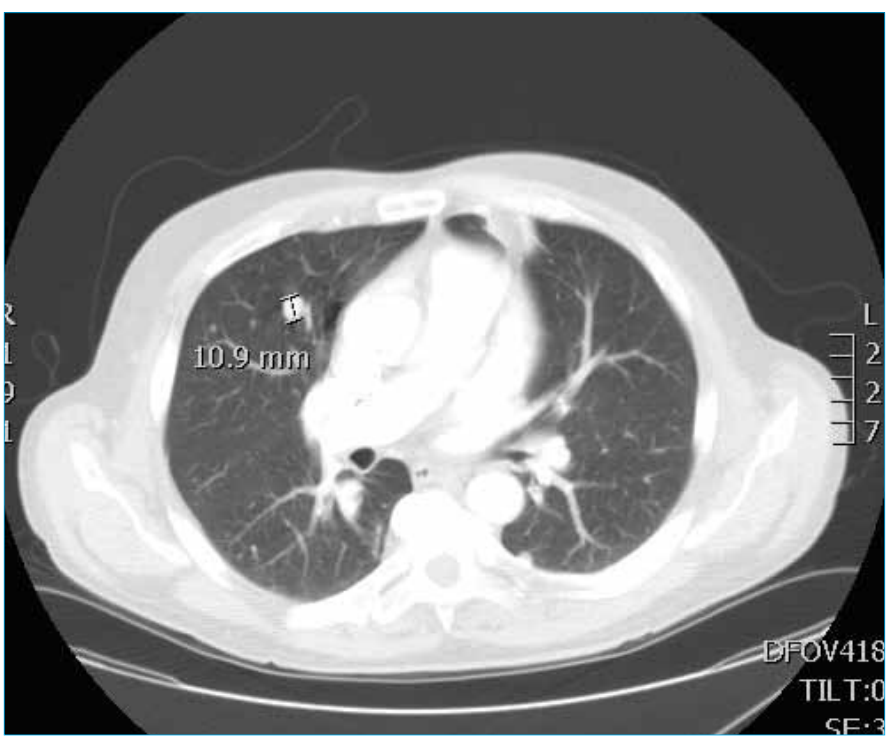

Fig. 4a. Pulmonary metastasis prior to cytoreductive nephrectomy.

tion, metastatic RCC to the testes was identified in patients between the ages of 45 and $85 .^{10}$

The clinical presentations of these cases vary; however, the most common method of discovery was the incidental finding of metastatic RCC in the orchiectomy pathology. ${ }^{6}$ The rarity of RCC spreading to the testis presents a unique challenge of accurately recognizing metastatic disease. As in our case, patients are often incorrectly assumed to have independent testicular disease. Given the fact that our patient was 68 , the probability of a primary germ cell tumour of the testicle was unlikely. Non-Hodgkin lymphoma remains the most commonly diagnosed testicular neoplasm in patients over $50 ;^{12}$ however, in a patient suspected of harbouring malignancy elsewhere in the body, the possibility of metastatic disease to the testicle must be ruled out. Even in those having been previously treated for renal malignancy, disease relapse has present as recurrences in the testicle. ${ }^{7}$ Thus, when evaluating an elderly patient with a testicular mass, the

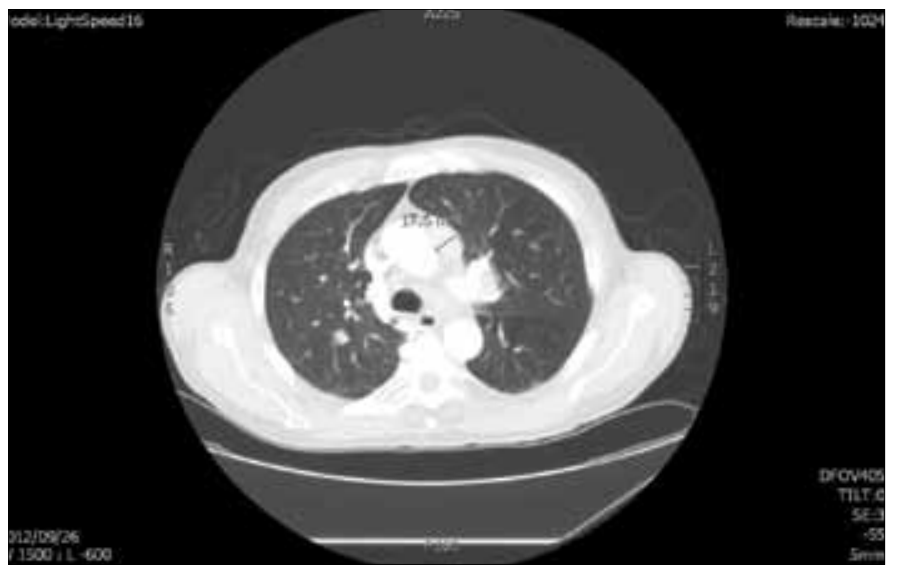

Fig. 5. Pulmonary metastasis 3 months after cytoreductive nephrectomy.

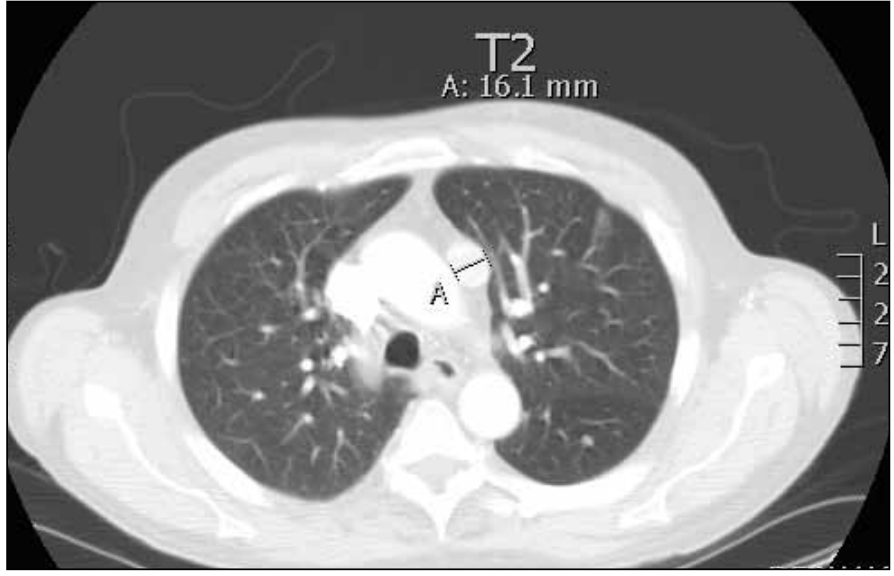

Fig. 4b. Pulmonary metastasis prior to cytoreductive nephrectomy.

possibility of metastatic disease must be considered in the differential diagnosis, especially in the setting of suspected or remote malignancy.

The exact method of spread of RCC to the testes is largely unknown, therefore adding to the difficulty of its recognition. One proposed explanation is through a mechanism of retrograde descent whereby tumour cells can directly seed via descent through the testicular veins. ${ }^{9}$ This theory appears to be most plausible for ipsilateral deposits of metastasis. However, this fails to explain how RCC lesions can spread to contralateral testicles. From our review of the available data, we found that there were at least 7 cases with contralateral involvement reported in the literature, including our case above. ${ }^{5-11}$ Even with many reports, the mechanisms of metastasis is still unclear; however, there are suggestions of spread via Batson's venous complex..$^{9,11,13}$ This avalvular venous network may provide the necessary conduit between contralateral renal capsular veins and spermatic cord ves-

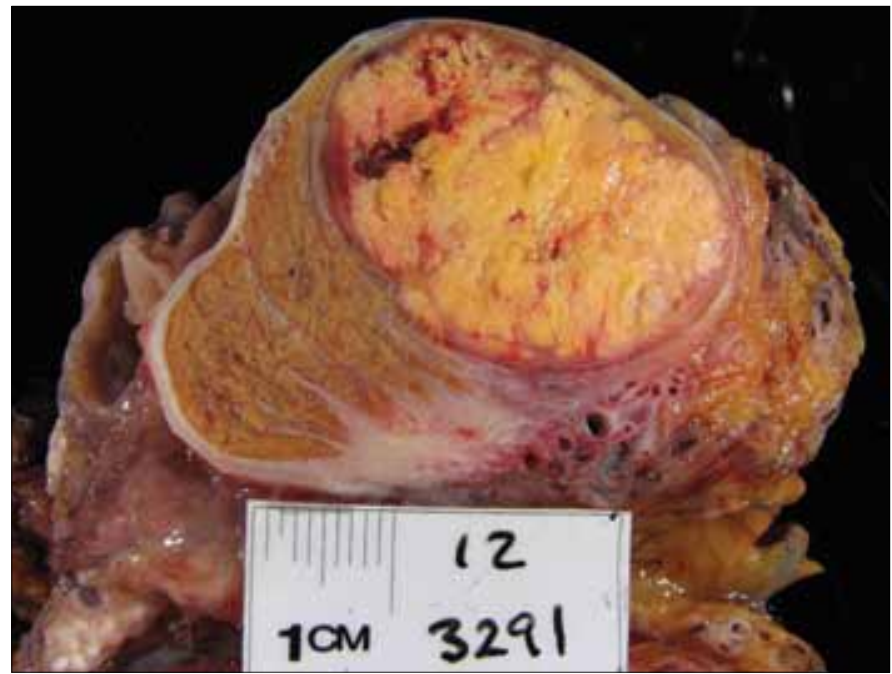

Fig. 6. Gross photograph showing well-circumscribed yellow and hemorrhagic mass confined to the testicle. 


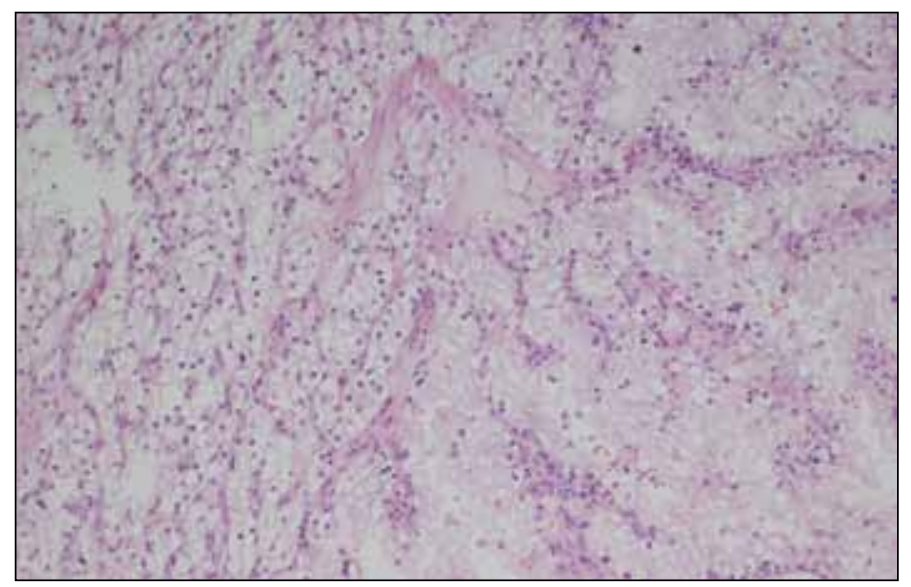

Fig. 7a. Renal cell carcinoma, clear cell type showing small nested and alveolar growth pattern with higher grade nuclear morphology on the right (hematoxylin and eosin 100x).

sels, thus potentially explaining distant seeding from a right RCC to a left testicle, as in our case above. Interestingly, we have not identified any reports of RCC spread to both testes simultaneously.

\section{Conclusion}

Although this case represents a rare entity of RCC metastatic spread to the testes, it serves as an important reminder for all physicians regarding the importance of a thorough genitourinary physical examination, which can often be overlooked in males. In our situation above, it was the patient's chief complaint that prompted the investigation; however had this not been the case, it may have potentially delayed the diagnosis of metastatic disease. Therefore, even when patients present with a seemingly unrelated issue, a thorough genitourinary and prostate examination should be routine in the primary assessment of the male patient.

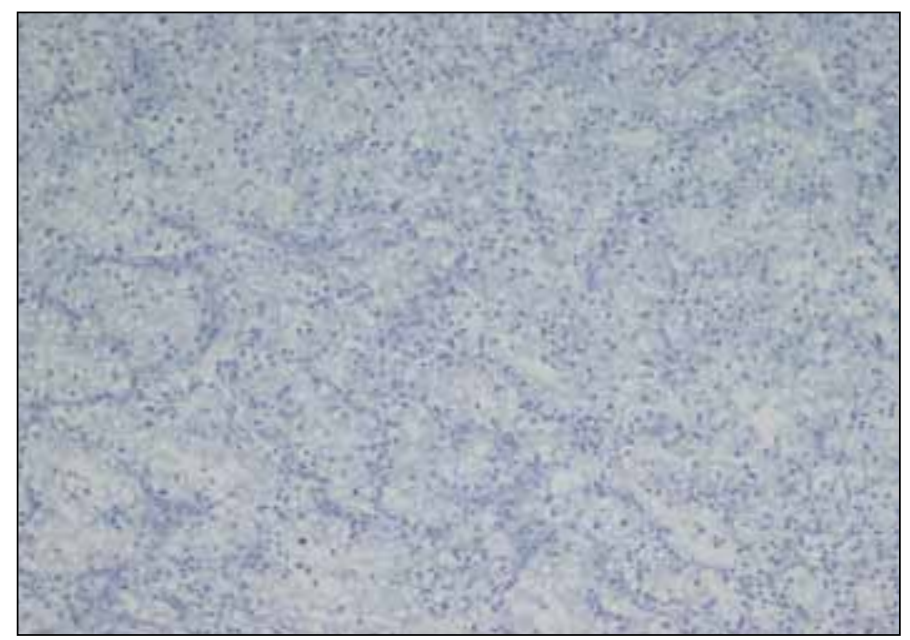

Fig. 7c. Renal cell carcinoma showing a negative result with cytokeratin 7 stain (CK7 immunostain; 100×).

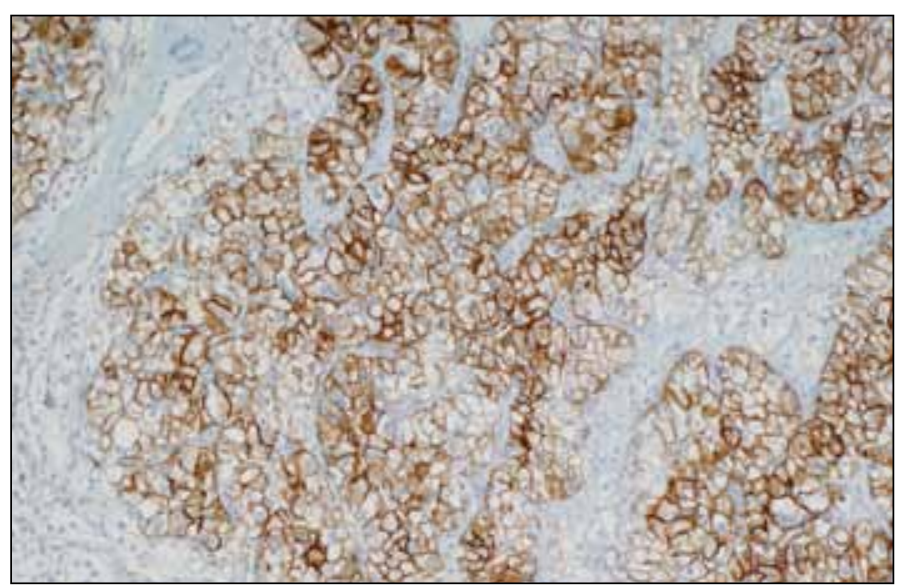

Fig. 7b. Renal cell carcinoma showing strong membranous staining with CD10 (CD10 immunostain; 100x).

Competing interests: Authors declare no competing financial or personal interests.

This paper has been peer-reviewed.

\section{References}

1. Jemal A, Siegel R, Ward E, et al. Cancer statistics 2006. CA Cancer I Clin 2006;56:106-30. http:// dx.doi.org/10.3322/caniclin.56.2.106

2. Gupta K, Miller JD, Li JZ, et al. Epidemiologic and socioeconomic burden of metastatic renal cell carcinoma (mRCC): A literature review. Cancer Treat Rev 2008;34:193-205. http://dx.doi.org/10.1016/i. ctrv.2007.12.001

3. Liungberg B, Campbell SC, Cho HY, et al. The epidemiology of renal cell carcinoma. Eur Urol 201 1;60:61521. http://dx.doi.org/10.1016/i.eururo.2011.06.049

4. Athar U, Gentile TC. Treatment options for metastatic renal cell carcinoma: A review. Can J Urol 2008;15:3954-66.

5. Dieckmann KP, Due w, Loy V. Intrascrotal metastasis of renal cell carcinoma. Case reports and review of the literature. Eur Urol 1988;15:297-301.

6. Ulbright TM, Young RH. Metastatic carcinoma to the testis. A clinicopathologic analysis of 26 nonincidental cases with emphasis on deceptive features. Am I Surg Pathol 2008;32:1683-93. http://dx.doi. org/10.1097/PAS.0b013e3181788516

7. Wu HY, Xu LW, Zhang YY, et al. Metachronous contralateral testicular and bilateral adrenal metastasis of chromophobe renal cell carcinoma: A case report and review of the literature. Biomed \& Biotechnol 2010;11:386-9.

8. Sountoulides P, Metaxa L, Cindolo L. Atypical presentations and rare metastatic sites of renal cell carcinoma: A review of case reports. J Med Case Reports 2011;5:429. http://dx.doi.org/10.1186/17521947-5-429

9. Steiner $\mathrm{G}$, Heimbach D, Pakos E, et al. Simultaneous contralateral testicular metastasis from a renal cell carcinoma. Scand J Urol Nephrol 1999;33:136-7. http://dx.doi.org/10.1080/003655999750016168

10. Datta MW, Ulbright TM, Young RH. Renal cell carcinoma metastatic to the testis and its adnexa: A report of five cases including three that accounted for the initial clinical presentation. Int I Surg Pathol 2001;9:49-56. http://dx.doi.org/10.1177/106689690100900108

11. Daniels GF, Schaeffer AJ. Renal cell carcinoma involving penis and testis: Unusual initial presentations of metastatic disease. Urology 1991;37:369-73. http://dx.doi.org/10.1016/0090-4295(91)80269-D

12. Stephenson AJ, Gilligan TD. Campbell-Walsh Urology. 10th ed. Philadelphia: PA. Wein; 2012.

13. Batson OV. The function of the vertebral veins and their role in the spread of metastases. Annals Surg 1940;112:138-49. http://dx.doi.org/10.1097/00000658-194007000-00016

Correspondence: Dr. Karim Marzouk, Department of Urology, Dalhousie University, Halifax, NS; kmarzouk@dal.ca 